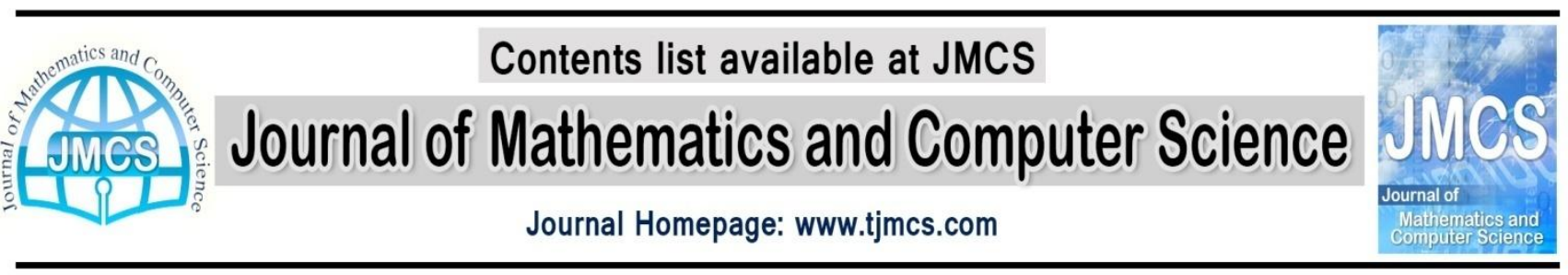

\title{
Survey Article about Image Fuzzy Processing Algorithms
}

\author{
Seyyed Mohammad Reza Hashemi ${ }^{1}$, Mohsen Zangian ${ }^{2}$, Mojtaba Shakeri ${ }^{3}$, Maryam Faridpoor ${ }^{4}$ \\ ${ }^{1}$ Young Researchers and Elite Club, Qazvin Branch, Islamic Azad University, Qazvin, Iran \\ ${ }^{2}$ Science and Research Branch, Islamic Azad University, Shahroud, Iran \\ ${ }^{3}$ Faculty of Computer and Information Technology Engineering, Qazvin Branch, Islamic Azad University, Qazvin, Iran \\ ${ }^{4}$ Science and Research Branch, Islamic Azad University, Semnan, Iran
}

Article history:

Corresponding Author: E-mail eng.reza2013@yahoo.com

Received July 2014

Accepted August 2014

Available online September 2014

\begin{abstract}
In this article, we reviewed the latest performed works in the image processing using fuzzy systems and methods. First, we introduced the fuzzy processing framework of the images and then study the application of fuzzy methods and systems in the main works of the image processing such as edge detection, image enhancement, segmentation and image description.
\end{abstract}

Keywords: edge detection, image enhancement, segmentation, image description, measuring the similarity and minimizing fuzzy degree.

\section{1- Introduction}

Definitions FCM Algorithm:

The conventional FCM algorithm divides a set of data $X=\left\{X_{1}, X_{2}, \ldots X_{n}\right\}$ into a number of $C$ clusters based on minimizing the following quadratic function.

$$
J_{F C M}=\sum_{i=1}^{c} \sum_{k=1}^{n} u_{i k}^{m} d_{i k}^{2}
$$

While the membership function $\mu_{i k} \varepsilon[0,1]$ represents the pixel degree $X_{k}(k=1,2, \ldots, n)$ that belongs to the cluster $\mathrm{i}(1 \leq i \leq c)$ and fuzzy parameter $m \varepsilon(1, \infty)$ determines the fuzzy value of the classification result and $d_{i k}=\left\|x_{k}-v_{i}\right\|$ is the distance between pixel $\mathrm{X}_{\mathrm{k}}$ and the centrality $\mathrm{V}_{\mathrm{i}}$ of the cluster $\mathrm{i}$. According to the definition of fuzzy theory, the membership functions limit each pixel that $\sum_{i=\gamma}^{c} \mu_{\mathrm{ik}}=$ 、 with this limitation of the objective function $U=\left\{\mu_{i k}\right\} \in R^{n \times c}$ can be a local 


$$
u_{i k}^{*}=\left\{\sum_{j=1}^{c}\left[\left(\frac{d_{i k}}{d_{j k}}\right)^{2 /(m-1)}\right]\right\}^{-1}: i=1, \ldots, c ; \quad k=1, \ldots, n
$$

minimum as following by updating the fuzzy membership function and the cluster centers $\mathrm{V}=\left\{\mathrm{V}_{1}, \mathrm{~V}_{2}, \ldots \mathrm{V}_{\mathrm{n}}\right\}$.

$$
v_{i}^{*}=\frac{\sum_{k=1}^{n} u_{i k}^{m} x_{k}}{\sum_{k=1}^{n} u_{i k}^{m}} ; \quad i=1, \ldots, c
$$

\section{2-1 Image Fuzzy Processing Framework}

An image $X$ with the dimensions of $M \times N$ and a gray level $L$ with the values of $g=0,1,2, \ldots, L-1$ can be defined as an array of Singleton fuzzy (a fuzzy set only with a Support point) that indicates the membership value of each pixel related to a pre-defined feature of the image. (For example: brightness, smoothness and the edge quality):

$$
X=\bigcup_{m=1}^{M} \bigcup_{n=1}^{N} \frac{\mu_{m n}}{g_{m n}} \quad \text { with } \mu_{m n} \in[0,1],
$$

As $g_{m n}$ and $\mu_{m n}$ indicate the fuzzy set membership symbol of $m, n^{\text {th }}$ pixels. The definition of the membership values is dependent on the special necessities of specific applications and the knowledge of the related expert person.

Fuzzy image processing has also 3 stages like other fuzzy methods: fuzzification $\phi$, suitable operation $\Gamma$ on the membership values and if necessary defuzzification $\psi$. The output $Y$ of the system for input $X$ is obtained by the following process chain:

$$
Y=\Psi(\Gamma(\Phi(X))) \text {. }
$$

The main difference of this method with other image processing methods is that the data input $X$ (histogram, gray levels and the feature) are processed on the membership plane. And this plane can use many varieties of fuzzy logic, fuzzy set theory and fuzzy measurement theory that can change or aggregate the membership values, data classification or decision making using the fuzzy inference. New membership values are changed again on the gray level so that histogram, varied gray levels, image segments or some classes of the new objects will be created. 


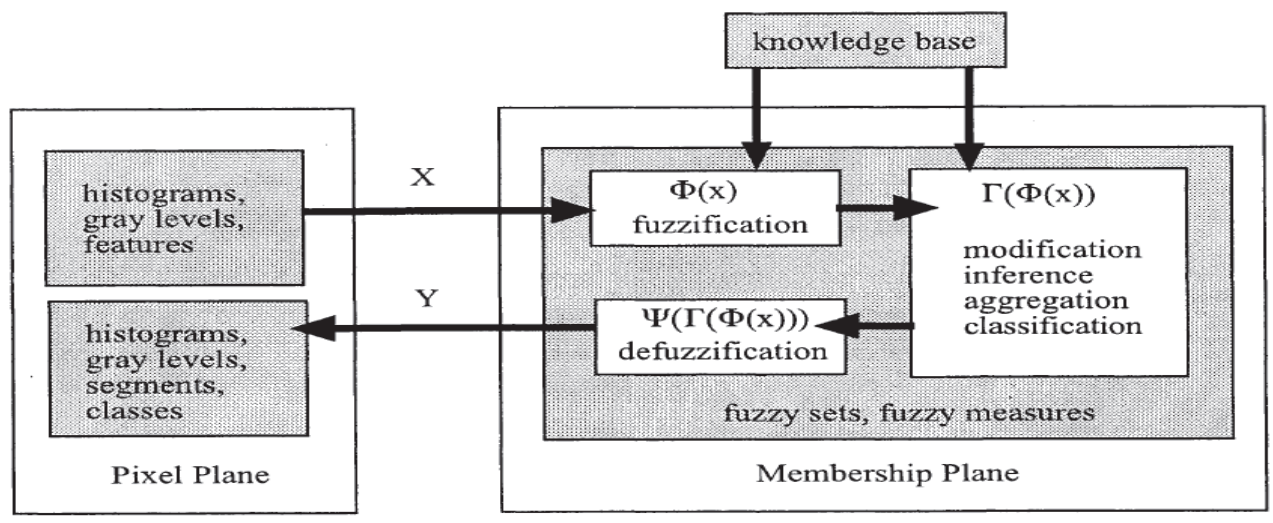

Figure 1: General structure of image fuzzy processing

Considering the image as digital fuzzy sets, we may need to have some information about measurement theory. Here a different size of the fuzzy quality has been offered so that they can find a quantitative answer for "How is fuzzy an image?"

Kaufmann has introduced the fuzzy quality index $\gamma$ of a fuzzy set $A \subseteq X$ as follows:

$$
\gamma(A)=\frac{2}{n^{k}} \times d\left(A, A^{\text {near }}\right)
$$

That $d$ is a suitable metric in the set $X, k$ is a positive real number and $n$, the number of the backup points. $A^{\text {near }}$ is the nearest Crisp set to A. using Minkowski q-norm as a metric and putting $k=\frac{1}{q}$, the fuzzification index can be defined as follows:

$$
\gamma(A)=\frac{2}{n^{\frac{1}{q}}}\left\{\sum_{i}\left|\mu_{A}\left(x_{i}\right)-\mu_{A^{n e a r}}\left(x_{i}\right)\right|^{q}\right\}^{\frac{1}{q}} .
$$

Minimizing the operator as a T-Norm, a linear fuzzification index $\gamma$ linear for an image A with the gray levels $\mathrm{g}$ and histogram $\mathrm{h}(\mathrm{g})$, the following expression is obtained:

$$
\gamma_{\text {linear }}(A)=\frac{2}{M N} \sum_{g=0}^{L-1} h(g) \cdot \min \left[\mu_{A}(g), \bar{\mu}_{A}(g)\right],
$$

Considering the space of image data, the image quality index can be rewritten as follows:

$$
\gamma_{\text {linear }}(A)=\frac{2}{M N} \sum_{i=1}^{N} \sum_{j=1}^{M} \min \left[\mu_{A}\left(g_{i j}\right), \bar{\mu}_{A}\left(g_{i j}\right)\right] \text {. }
$$


Other fuzzy quality units are quadratic fuzzy quality index $\gamma$ quadratic, entropy fuzzy Hs and Exponential fuzzy Hexp:

$$
\gamma_{\text {quadratic }}(A)=\frac{2}{\sqrt{M N}} \sqrt{\sum_{g=0}^{L-1}\left[h(g) \times \min \left[\mu_{A}(g), \bar{\mu}_{A}(g)\right]\right]^{2}},
$$

These measurements are usually used during the improvement, getting threshold and classification.

\section{Materials and Methods}

\section{2-2 Image Fuzzy Edge Detection}

M.Emim has used a new Neuro-Fuzzy operator to detect the edge in the digital images. The proposed operator has been made of a combination of a sub detector with a post processor. Each NF sub detector in the structure evaluates a pixel in a neighborhood relationship. Therefore, the number of NF sub detectors in the structure may be variable to obtain the desired edge detection efficiency. The internal parameters of NF sub detector are optimized using the artificial image learning.

Neuro-Fuzzy sub detector: each NF sub detector is a first order fuzzy interference system from the kind of Sugeno that has 3 inputs and 1 output. The internal structures of NF sub detectors are equal. Each input has three membership functions from the kind of Generalized Bell and each output has one linear membership function. The input - output relationship of each of NF sub detectors is as follows:

Assuming $x 1, X 2$ and $X 3$ denote the input of NF sub detector and $Y$ specifies the output, every possible combination of the inputs and related membership functions is shown by a rule in the rule base of NF sub detector. Since NF sub detector has three inputs and each input has three membership functions, the rule base contains totally $27\left(2^{3}\right)$ rules as follows:

1. if $\left(X_{1}\right.$ is $\left.M_{11}\right)$ and $\left(X_{2}\right.$ is $\left.M_{21}\right)$ and $\left(X_{3}\right.$ is $\left.M_{31}\right)$, then $R_{1}=F_{1}\left(X_{1}, X_{2}, X_{3}\right)$

2. if $\left(X_{1}\right.$ is $\left.M_{11}\right)$ and $\left(X_{2}\right.$ is $\left.M_{21}\right)$ and $\left(X_{3}\right.$ is $\left.M_{32}\right)$, then $R_{2}=F_{2}\left(X_{1}, X_{2}, X_{3}\right)$

3. if $\left(X_{1}\right.$ is $\left.M_{11}\right)$ and $\left(X_{2}\right.$ is $\left.M_{21}\right)$ and $\left(X_{3}\right.$ is $\left.M_{33}\right)$, then $R_{3}=F_{3}\left(X_{1}, X_{2}, X_{3}\right)$

4. if $\left(X_{1}\right.$ is $\left.M_{11}\right)$ and $\left(X_{2}\right.$ is $\left.M_{22}\right)$ and $\left(X_{3}\right.$ is $\left.M_{31}\right)$, then $R_{4}=F_{4}\left(X_{1}, X_{2}, X_{3}\right)$

5. if $\left(X_{1}\right.$ is $\left.M_{11}\right)$ and $\left(X_{2}\right.$ is $\left.M_{22}\right)$ and $\left(X_{3}\right.$ is $\left.M_{32}\right)$, then $R_{5}=F_{5}\left(X_{1}, X_{2}, X_{3}\right)$

27. if $\left(X_{1}\right.$ is $\left.M_{13}\right)$ and $\left(X_{2}\right.$ is $\left.M_{23}\right)$ and $\left(X_{3}\right.$ is $\left.M_{33}\right)$, then $R_{27}=F_{27}\left(X_{1}, X_{2}, X_{3}\right)$,

While, $M_{i j}$ specifies $\mathrm{i}^{\text {th }}$ membership function $\mathrm{i}^{\text {th }}$ input, $R_{\mathrm{k}}$ is the output of $\mathrm{k}^{\text {th }}$ rule and $\mathrm{F}_{\mathrm{k}}$ is $\mathrm{k}^{\text {th }}$ output of the membership function. The input membership function from the kind of Generalized Bell is as follows: 


$$
M_{i j}(u)=\frac{1}{1+\left|\frac{u-a_{i j}}{b_{i j}}\right|^{2 c_{i j}}}
$$

And output membership function is linear and as follows:

$$
F_{k}\left(u_{1}, u_{2}, u_{3}\right)=d_{k 0}+d_{k 1} u_{1}+d_{k 2} u_{2}+d_{k 3} u_{3}
$$

\section{Proposed Neuro-Fuzzy Operator:}

Figure 1 shows the general picture of the proposed structure for the edge detection operator. All NF sub detectors in the structure work on a window filter which has been shown in figure 2. Each NF sub detector evaluates a different neighborhood relationship between window filter pixel center and its two neighbors. Some possible topologies of the neighborhoods are shown in Figure 3. 


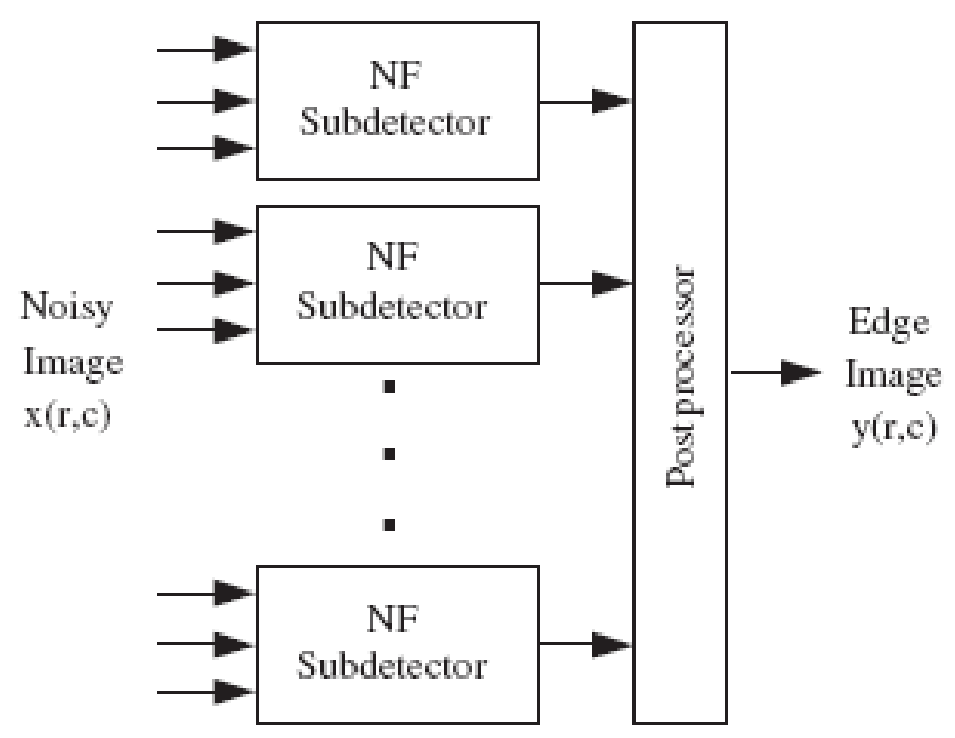

(a)

\begin{tabular}{|c|c|c|}
\hline$x(r-1, c-1)$ & $x(r-1, c)$ & $x(r-1, c+1)$ \\
\hline$x(r, c-1)$ & $x(r, c)$ & $x(r, c+1)$ \\
\hline$x(r+1, c-1)$ & $x(r+1, c)$ & $x(r+1, c+1)$ \\
\hline
\end{tabular}

(b)
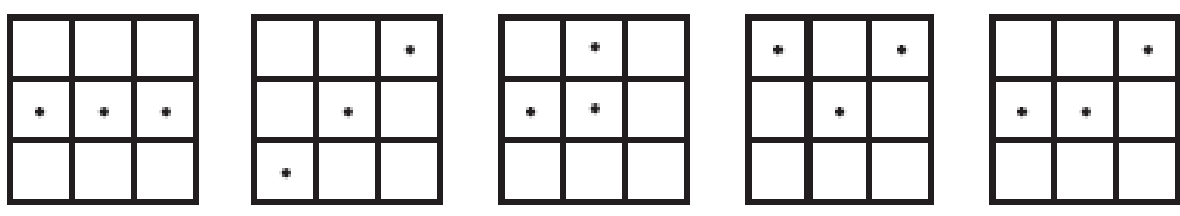

(c)

Figure 2: Neuro-Fuzzy general structure 

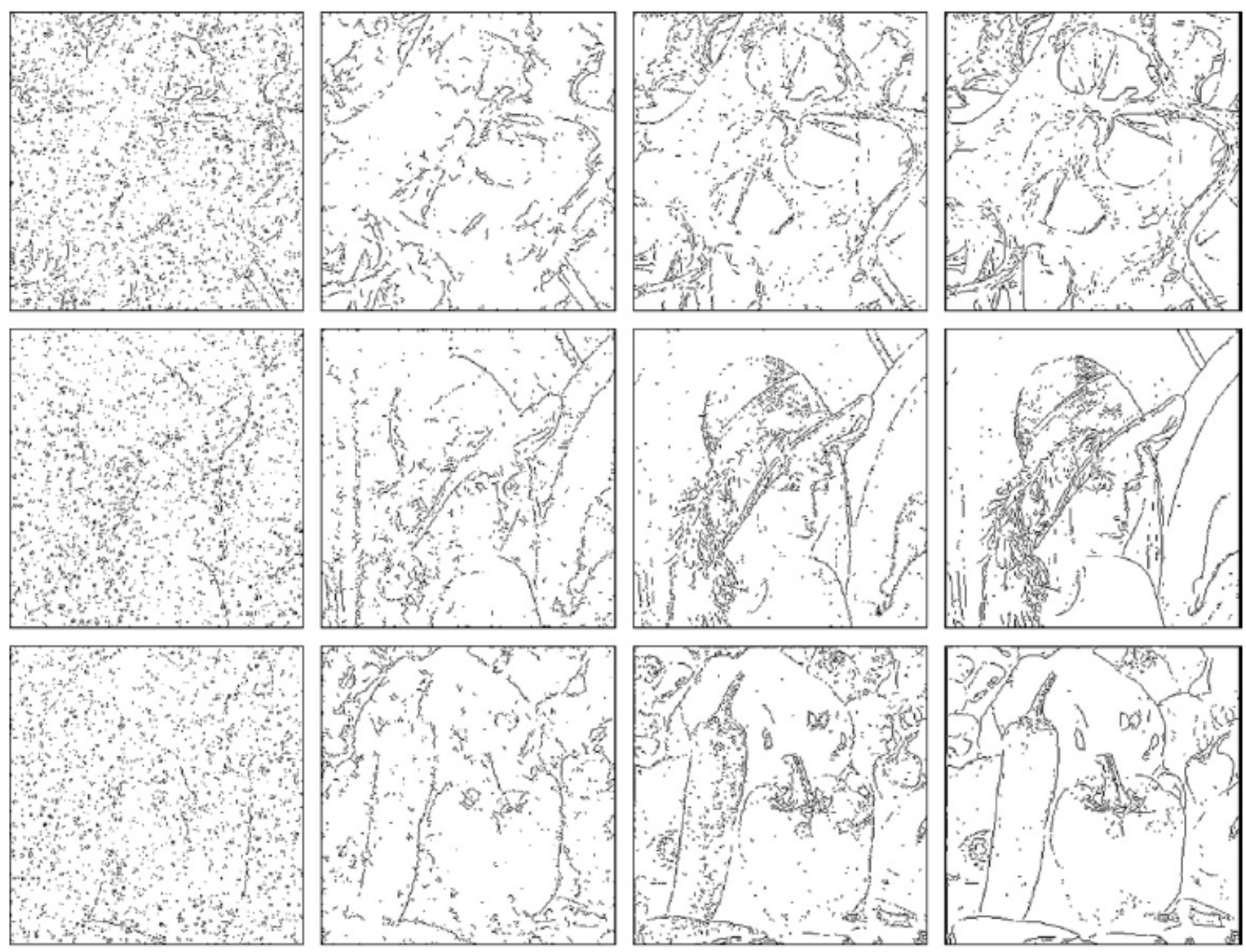

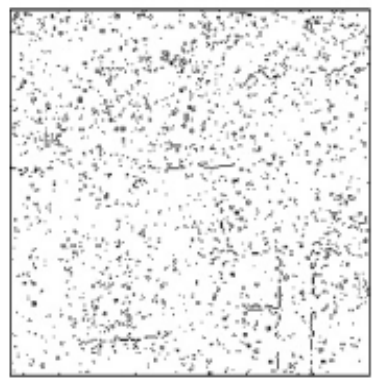

Sobel

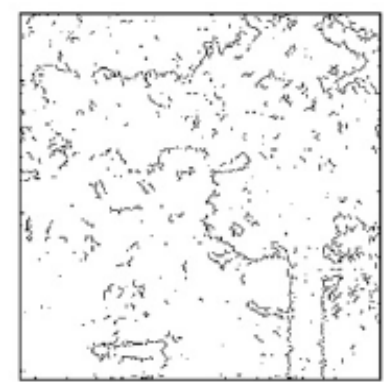

Canny

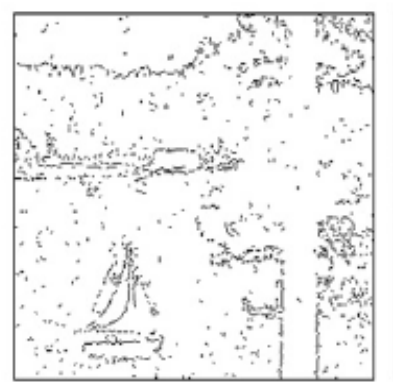

Proposed (2 NF subdet.)

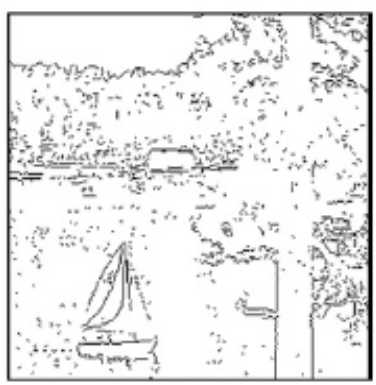

Proposed (8 NF subdet.)

Figure 3: Comparison between the proposed method and other methods

C.Lopez-Molina and et al have proposed a method that considered the membership functions of the different parameters to convert the gradient into a membership degree. Then one strategy based on the histogram has been introduced to determine the values of these parameters automatically to conform the membership functions to some characteristics of each image.

\section{Gradient Membership Functions:}

Working with gray images, one gradient is shown by horizontal and vertical components. Therefore, we need the following mapping in order to convert the gradient of an image into the membership degree: 


$$
g: \mathbb{R}^{2} \rightarrow[0,1]
$$

In any way, in a general purpose of edge detection method, the gradient directions don't make a difference. So we have considered the function $g$ to work on the Euclidean $g: \mathbb{R}^{+} \rightarrow[0,1]$ domain of the gradient:

\section{3- Fuzzy Enhancement of Images}

\section{3-1 Similarity Measurement}

Many criteria that represent the similarity or equality between two fuzzy sets can be found in the literature. Here there is no unique definition; most often they are used as follows. A similarity criterion is a fuzzy binary relation in $F(k)$, i.e. $(K) \times F(K)->[0,1]$ which its mapping is reflective, symmetrical and trespass.

\section{3-1-1 creating a Similarity Measurement based on the Neighborhood}

In articles, many measures have been proposed for the expression of similarity between two fuzzy sets. In a set with more than 40 similarity criteria, 14 similarity measurements will provide abovementioned characteristics if they are applied directly for above images. Among them, 8 similarity measurements are appropriate to compare the images. We want to discuss about all similarity measurement lists but we want to recollect the expression of several examples about similarity measures briefly.

The first similarity measurement based on Minkowski distance and observing the lesser distance between $A$ and $B$ has the most similarity between $A$ and $B$. These observations lead to the following similarity criteria for $\mathrm{M} 1$ and $\mathrm{M} 2$ that are $(r->\infty)$

$$
\begin{aligned}
M_{1}(A, B)= & 1-\left(\frac{1}{M N} \sum_{(x, y) \in X}|A(x, y)-B(x, y)|^{r}\right)^{\frac{1}{r}} \\
& \text { with } r \in \mathbb{N} \backslash\{0\} \\
M_{2}(A, B)= & 1-\max _{(x, y) \in X}|A(x, y)-B(x, y)| .
\end{aligned}
$$

We need a notice for fuzzy set cardinality to measure next similarities. The cardinality of Crisp limited set becomes known by a number of the elements in that set. This concept can spread in fuzzy sets using Sigma Count. Sigma Count is defined in community $\mathrm{X}$ as follows:

$$
|A|=\sum_{x \in X} A(x)
$$

The following similarity measurements are based on Cardinality: 


$$
\begin{aligned}
M_{6}(A, B) & =\frac{|A \cap B|}{|A \cup B|} \\
& =\frac{\sum_{(x, y) \in X} \min (A(x, y), B(x, y))}{\sum_{(x, y) \in X} \max (A(x, y), B(x, y))} \\
M_{7}(A, B) & =\frac{|A \cap B|}{\max (|A|,|B|)} \\
& =\frac{\sum_{(x, y) \in X} \min (A(x, y), B(x, y))}{\max \left(\sum_{(x, y) \in X} A(x, y), \sum_{(x, y) \in X} B(x, y)\right)}
\end{aligned}
$$

Finally, we will mention the following similarity measurements which are useful for comparing the images:

$$
M_{20}(A, B)=\frac{1}{M N} \cdot \sum_{(x, y) \in X}\left[\frac{\min (A(x, y), B(x, y)}{\max (A(x, y), B(x, y))}\right]
$$

\section{3-2- Similarity Measure for Digital Image Histogram}

In this section, we review briefly to make the similarity measures based on the neighborhood, which are also combined each other homogenously in the different neighborhoods. We start this by calculating the similarity between the separate parts of the image between two images $A$ and $B$. So, we divide two images $A$ and $B$ into $8 \times 8$ separate parts and calculate the similarity between each of the parts of $8 \times 8$ image. To calculate the similarity between the parts of two images, we simply apply the pixel-based similarity criteria to the parts of both images. Assuming the image is divided into $n$ segments and the similarity between the part $A i$ of the image $A$ and part $B i$ of the image $B$ has been determined by $M(A i, B i)$, the similarity between two images $A$ and $B$ is obtained by the mean of similarities of the related separated sections of the image. Therefore we have:

$$
M^{h}(A, B)=\frac{1}{N} \sum_{i=1}^{N} w_{i} \cdot M\left(A_{i}, B_{i}\right)
$$

However, the similarity of $\mathrm{M}(\mathrm{Ai}, \mathrm{Bi})$ was limited using the similarity criteria to the segments of the images $\mathrm{Ai}$ and $\mathrm{Bi}$ and the weight $\mathrm{Wi}$ is defined as the similarity between the homogeneity hAi of the segment of the image $i$ in the image $A$ and the homogeneity $h B i$ of the segment of the image $i$ in the image $B$. The homogeneity hAi of the segment of the image $A$ is calculated as the similarity between the gray values of the pixel in the segment of the image with the highest intensity and the gray value in the segment of the image with the lowest intensity is calculated using the similarity relation. Therefore we have:

$$
\begin{aligned}
h_{A_{i}} & =s\left(\max _{(x, y) \in A_{i}} A(x, y), \min _{(x, y) \in A_{i}} A(x, y)\right) \\
w_{i} & =s\left(h_{A_{i}}, h_{B_{i}}\right) .
\end{aligned}
$$




\section{3-2- Contrast / Brightness}

Contrast and brightness are the features of the image so, if pre-process is necessary for human comprehension, it should be done (for example in medical applications). Mainly there are two ways to adjust the contrast / brightness of the image: changing the gray level with an appropriate function F:

$$
g^{\prime}=f(g)
$$

or the operation on the histogram. The Efficiency of the first method depends on the figure of the selected function that some automatic methods have been presented to select the appropriate function. The second method is to change the image histogram that Histogram Equalization is one of the best known techniques.

The improvement of the gray level $\mathrm{g}$ is calculated by the following equation:

$$
g^{\prime}=f_{h}(g)=(L-1) \sum_{i=0}^{g} \frac{h(i)}{M N},
$$

However, the right side of the expression should be approximated to obtain an integer number for $\mathrm{g}$.

\section{3-2-1- Fuzzy Degree Minimization}

This method is probably the first fuzzy approach for the image enhancement and has been recognized as a contrast intensifier operator in the articles. The main idea of this method is to reduce some of the image fuzzy quality. The overall algorithm can be formulated by the following expression:

The fuzzification of the gray level ( $\mu(\mathrm{g})$ denotes the degree of brightness)

$$
\mu(g)=\left[1+\frac{g_{\max }-g}{F_{d}}\right]^{-F_{e}}
$$

The improvement of the membership degree using the successive application of the Zadeh intensification operator

$$
\mu^{\prime}(g)=\left\{\begin{array}{lll}
2[\mu(g)]^{2} & \text { if } & 0 \leq \mu(g) \leq 0.5 \\
1-2[1-\mu(g)]^{2} & \text { if } & 0.5<\mu(g) \leq 1
\end{array}\right.
$$

Making new gray levels by Deffuzification

$$
g^{\prime}=g_{\max }-F_{d}\left(\left(\mu^{\prime}(g)\right)^{\frac{-1}{F_{e}}}-1\right), \quad\left(1+\frac{g_{\max }}{F_{d}}\right)^{-F_{e}} \leq \mu^{\prime}(g) \leq 1
$$

Fe and Fd are exponential and cluster fuzzifications that control the value of being gray haze in the membership plane.

\section{3-2-2- Making Equivalent U fuzzy Expected Values}


In this approach, Fuzzy Expected value (FEV) or a Weighted Version (WFEV) has been calculated to enhance the image quality related to all gray levels of FEV.

A fuzzy relation in a universal set $X$ produces a fuzzy set $\mathrm{F}$ with the membership function $X_{f}$ and $0 \leq \chi_{F}(x) \leq 1, x \in X$. Having $\xi_{T}=\left\{x \mid \chi_{F}(x) \geq T, T \in[0,1]\right\}$ as a subset and $\mu$, a defined fuzzy measure in the subsets $F$ is:

$$
\mu\left(\xi_{T}\right)=\frac{N_{x: \chi_{F}(x) \geq T}}{N}
$$

However, $\mathrm{N}$ is the total number of the elements. Fuzzy Expected Value $X_{f}$ on $\mathrm{F}$ is defined as follows:

$$
\mathrm{FEV}=\sup _{0 \leq T \leq 1}\left\{\min \left[T, \mu\left(\xi_{T}\right)\right]\right\}
$$

The algorithm of this method is formulated as follows:

1- The calculation of the image histogram

2- The calculation of the most common value ( $\mathrm{K}=\mathrm{FEV}) \mathrm{K}$

3- The calculation of the distance $D$ of each gray level

$$
D=\sqrt{\left|K^{2}-g^{2}\right|}
$$

4- The production of new gray levels g'

$$
g^{\prime}= \begin{cases}\max (0, K-D) & \text { if } g<M \\ \min (L-1, K+D) & \text { if } g>M \\ K & \text { otherwise }\end{cases}
$$

\section{3-2-3- Contrast Enhancement based on the Rules}

The fuzzy approach based on the rule is a powerful general method for some tasks in the image processing. Its algorithm is as follows:

- Preparing the inference system parameters (number of input and output membership functions, the shapes and positions)

- The fuzzification of gray levels (for example, the membership degree values of dark, gray and lighter sets than gray levels)

. The inference approach of evaluating the appropriate rules (For example, if dark, then black, if gray, then gray and if light, then white)

. Output defuzzification using 3 singletons (for example):

$$
g^{\prime}=\frac{\mu_{\text {dark }}(g) \times S_{1}+\mu_{\text {gray }}(g) \times S_{2}+\mu_{\text {bright }}(g) \times S_{3}}{\mu_{\text {dark }}(g)+\mu_{\text {gray }}(g)+\mu_{\text {bright }}(g)} .
$$




\section{3-2-4- Enhancement based on Fuzzy Relations}

Bhutani and Battou have been used the fuzzy relations to develop a new enhancement technique. Considering an image with $\mathrm{M}$ lines and $\mathrm{N}$ columns, you can see the image as a weighted relation from a set $\mathrm{X}$ with $\mathrm{M}$ elements of the set $\mathrm{Y}$ with $\mathrm{N}$ elements. While $g_{i, j}$ denotes the weight in the place $(\mathrm{i}, \mathrm{j})$. The image can be defined as a fuzzy relation $\mu$ from $X$ to $Y$ with the membership degree $\mu_{i, j}$ in each place $(i$, j) as:

$$
\mu_{i j}= \begin{cases}0 & \text { if } \quad g_{i j}<T_{1}, \\ 0.5\left(\frac{g_{i j}-T_{1}}{T-T_{1}}\right) & \text { if } \quad T_{1} \leq g_{i j} \leq T, \\ 0.5\left(\frac{T_{2}-g_{i j}}{T_{2}-T}\right)+\left(\frac{g_{i j}-T}{T_{2}-T}\right) & \text { if } \quad T<g_{i j} \leq T_{2}, \\ 1 & \text { if } \quad g_{i j}>T_{2}\end{cases}
$$

While the threshold $\mathrm{T}$ (the mean gray level of the segment) $\mathrm{T} 1<\mathrm{T}$ (the mean gray level of the pixels with gray level lesser than T) and T2>T (the mean gray level of pixels with gray level greater than $\mathrm{T}$ ) can be defined by the user.

Fuzzy sets are defined for each segment of the image (for example: the object and the background) as follows:

$$
\begin{gathered}
\sigma_{\min X}(a)=\min _{y \in Y} \mu_{a y}, \quad \sigma_{\min Y}(b)=\min _{x \in X} \mu_{x b}, \\
\sigma_{\max X}(a)=\max _{y \in Y} \mu_{a y}, \quad \sigma_{\max Y}(b)=\max _{x \in X} \mu_{x b},
\end{gathered}
$$

As the minimum and maximum are defined in fuzzy values $\left(0<\mu_{i, j}<1\right)$

\section{3-3- Fuzzy Filter}

Image filtering is necessary for the enhancement of the edges and / or noise reduction. There are old various techniques that do this. The main problem of filtering is that adjusting the image characteristics such as sharpness and softness is difficult. Omitting the noise in an image, the finer details are also filtered. To enhance the edges and smooth out the structure, on the other hand they noises are also reinforced. Many methods have been developed to resolve these conflicts. A comprehensive approach is an incompatible filter that the image pixels $g_{m n}$ in each $\mathrm{N} \times \mathrm{N}$ neighborhoods ( $\mathrm{N}$ is an odd number) are attributed with a suitable weight $W_{i, j}$. The result of this filter can be obtained by the following equation: 


$$
g_{m n}^{\prime}=\frac{\sum_{i} \sum_{j} w_{m+i, n+j} \cdot g_{m+i, n+j}}{\sum_{i} \sum_{j} w_{m+i, n+j}}
$$

In recent years, many fuzzy methods have been proposed for filtering the image, which can be divided into the following categories:

. Net Fuzzy Filter: uses only if-then fuzzy properties

- Fuzzy Expansion of the Existing Algorithms: expands the old filters to fuzzy sets using the membership functions or fuzzy rules.

- Fuzzy Combination Techniques: collect the results of different filters to combine their advantages.

\section{4- Fuzzy Segmentation of the Images}

Segmentation is a main issue to describe the image classification. It is usually defined based on a same definition which is dependent on a particular work. This process refers to the division of an image into several regions which are connected together so that these regions don't overlap each other.

Many developed segmentation approaches can't be generalized in a certain order. According to the source [3 ], most approaches are based on the similarities and differences and in particular can be divided into the following categories: getting threshold, clustering, edge detection and region extraction.

Gour and Laurence who have used fuzzy rules based image segmentation algorithm (GFRIS), are independent in its application and use inter-pixel relational space. GFRIS algorithm estimates automatically both factors of the weight and threshold value respectively in the definition of fuzzy rule and neighborhood system respectively.

Two of the most popular fuzzy clustering techniques are PCM and FCM, while both of these methods have been widely applied; none of these hasn't integrated human experience knowledge and doesn't also include some information about the pixel relational space. Image segmentation that relies on some features based on the information without considering the inter-pixel relationships, generally don't produce good results because a large number of pixels usually overlap in different areas.

GFRIS algorithm Details can be expressed as follows:

1- To classify the pixels of an image into the regions with the required numbers using each clustering algorithm.

2- Key weight interference and the threshold value by applying data mining algorithms and the membership functions for the distribution of each pixel.

3- The preparation of the center of all areas should define the membership functions with the ratio of the produced centers by clustering algorithm in the first stage.

4- To select an uncategorized pixel of the image and to calculate each membership function value for that pixel in the area respectively.

5- Pixel classification to an area during the performance 
6- To Return Step 4 until all pixels are classified.

Zexuan and et al have been proposed an algorithm called the weighted image based on patch FCM (WIPFCM) for image segmentation. They used the image patches in this algorithm to replace the pixels in fuzzy clustering and to create a weighted sequence so that the pixels are able to have different weights in each image patch. The proposed algorithm combines the spatial information embedded in the image with the segmentation process and so makes it stronger than the noise.

Zhimin and et al have presented a dedicated fuzzy clustering algorithm with the space of information theory to improve the power of conventional clustering algorithms for image segmentation. Their method is to combine an information theory framework and FCM algorithm.

Combining these two concepts and modifying the objective function of FCM algorithm, we are able to solve the problems that are sensitive to the noise data and don' have the spatial data.

In image segmentation, the neighborhood pixels have more relationships in the time domain. If the segmentation algorithm has an error to calculate the neighborhood relationship, the segmentation result will have the fault and error.

In other words, data clustering algorithm should combine the spatial data in such a way that if neighborhood pixels share their similar characteristics, central pixel should have a high probability of grouping for the same clusters as neighborhood pixels.

Improving the objective function is one of the common ways used to combine the spatial data and the FCM algorithm.

Many researchers have changed the objective function to use the advantages of the central pixel. The general modified objective function can be expressed as follows:

However, $N_{i}$ is a subset of the pixels in the neighborhood $x_{\mathrm{i}}$ and the parameter $\alpha$ reflects the effective neighborhood reference. $\alpha$ can be constant in real applications and or considered for constant image content.

With this modification, the spatial data can be combined with the clustering process. However, the modification of above objective function may smooth the main structure in the image (such as surrounding areas or edges), because it always requires a significant amount of the limitation of the central pixel. In other words, the center pixel which is influenced by neighborhood pixels isn't important in a homogenous area or along the edge.

\section{5- Conclusions}

Fuzzy techniques offer a new and flexible framework for developing image processing algorithms. The methods are nonlinear and strong and based on the knowledge.

In comparison with the image processing, Fuzzy Set Theory Potential hasn't been studied as good as the certain methodologies. Therefore, one of the main objectives of this is the full search of each fuzzy approach. Information theoretical approaches that use some of the image fuzzification are too fast, while if rule-based techniques use image information space they will be computationally expensive. 


\section{References}

[1] C. Lopez-Molina, B. De Baets, H. Bustince, Generating fuzzy edge images from gradient magnitudes (Computer Vision and Image Understanding Volume 115, Issue 11, November 2011, Pages 1571-1580)

[2] Zexuan Ji a,*, Yong Xiab,c, Qiang Chena, Quansen Suna, Deshen Xiaa, David Dagan Feng, Fuzzy c-means clustering with weighted image patch for image segmentation (Applied Soft Computing Volume 12, Issue 6, June 2012, Pages 1659-1667)

[3] Zhimin Wanga,1, Qing Song b, , Yeng Chai Soh b, Kang Sim, An adaptive spatial information-theoretic fuzzy clustering algorithm for image segmentation (Computer Vision and Image Understanding Volume 117, Issue 10, October 2013, Pages 1412-1420)

[4] Gour C. Karmakar *, Laurence S. Dooley, A generic fuzzy rule based image segmentation algorithm (Pattern Recognition Letters Volume 23, Issue 10, August 2002, Pages 1215-1227)

[5] Mancuso M., Poluzzi R. and Rizzotto G.G., A fuzzy filter for dynamic range reduction and contrast ( Conference Publications > Fuzzy Systems, 1994. IEEE )

[6] Hamid R. Tizhoosh, Fuzzy Image Enhancement An Overview (Fuzzy Techniques in Image Processing Studies in Fuzziness and Soft omputing Volume 52, 2000, pp 137-171)

[7] L. Cinque, G. Foresti, L. Lombardi , A clustering fuzzy approach for image segmentation (Pattern Recognition 37 (2004) 1797 - 1807)

[8]L. A. Zadeh "A Computational Approach to Fuzzy Quantifiers in Natural Languages", (Computer and Mathematics, vol. 9, no. 1, pp.149-184 1983 )

[9] Bhutani K.R. and Battou A., An application of fuzzy relations to image enhancement,Pattern Recognition Letters, Vol. 16, pp. 901-909, 1995 Bouchon-Meunier B., “Aggregation and Fusion of Imperfect Information”, ( Physica-Verlag, Heidelberg, New York, 1998)

[10] N. Pal, S. Pal, A review on image segmentation techniques,(Pattern Recognition 26 (9) (1993) 1277-1294.)

[11] T. Peli, D. Malah, A study of edge detection algorithms,(Comput. Graphics Image process. 20 (1982) 